\title{
NOTE ON SYMBOLIC POWERS AND GOING DOWN
}

\author{
S. MCADAM AND L. J. RATLIFF, JR. ${ }^{1}$
}

\begin{abstract}
For primes $P \subseteq Q$ of a Noetherian ring $R$, we consider when, for all $k \geq 1$, there is an $m$ with $P^{(m)} \subseteq Q^{(k)}$, and reprove a relevant theorem of Schenzel. If $R$ is a domain, we consider sufficient conditions for $P \subseteq Q$ to satisfy going down for all primes $Q$ containing $P$.
\end{abstract}

Introduction. Let $P$ be a prime ideal in a Noetherian ring $R$. In [7], Schenzel shows that $E(P)$, the set of essential prime divisors of $P$ (defined below), equals $\{P\}$ if and only if the $P$-adic and $P$-symbolic topologies are equivalent. In the first section, we offer a new, and fairly easy proof. Our arguments shed light on when, for primes $P \subseteq Q$, for all $k \geq 1$, there is an $m$ with $P^{(m)} \subseteq Q^{(k)}$. The second section considers a prime $P$ in a Noetherian domain $R$, and shows that if $E(P)=\{P\}$, then $P \subseteq Q$ satisfies going down for all primes $Q$ containing $P$. In $\S 3$, we list some circumstances which assure that $E(P)=\{P\}$.

\section{Symbolic powers.}

Notation. $R$ will always be a Noetherian ring. Its integral closure will be denoted $R^{\prime}$, and if it is local, its completion will be $R^{*}$. If $I$ and $J$ are ideals of $R$, then the eventual stable value of $(I: J) \subseteq\left(I: J^{2}\right) \subseteq \cdots$ will be denoted by $I:\langle J\rangle$. For $I$ an ideal in $R, A^{*}(I)=$ Ass $R / I^{n}$ for all large $n$, while $\bar{A}^{*}(I)=$ Ass $R /\left(I^{n}\right)_{a}$ for all large $n,\left(I^{n}\right)_{a}$ denoting the integral closure of $I^{n}$. Finally, $E(I)=\{P \in \operatorname{Spec} R \mid I \subseteq P$ and there is a $z \in$ Ass $R_{P}^{*}$ with $P_{P}^{*}$ minimal over $\left.I R_{P}^{*}+z\right\}$.

$A^{*}(I)$ and $\bar{A}^{*}(I)$ are discussed in [2, Chapters 1 and 3], where it is shown that both are well defined finite sets. $E(I)$ is discussed in [4]. In our first lemma we list the facts about $E(I)$ which we need.

LEMMA 1.1. Let $I$ be an ideal in $R$.

(i) $E(I) \subseteq A^{*}(I)$.

(ii) If $Q$ is a prime containing $I$, then $Q \in E(I)$ if and only if there is an integer $k \geq 1$ such that, for all $m \geq 1, I^{m}:\langle Q\rangle \nsubseteq Q^{(k)}$.

(iii) If the prime $P$ is minimal over $I+z$, with $z \in$ Ass $R$, then $P \in E(I)$.

(iv) If $P \in \operatorname{Spec} R$ and $S$ is a multiplicatively closed subset of $R$ disjoint from $P$, then $P \in E(I)$ if and only if $P_{S} \in E\left(I_{S}\right)$.

(v) If $R \subseteq T$ is a finite integral extension of Noetherian domains, then $P \in E(I)$ if and only if there is $a \in E(I T)$ with $Q \cap R=P$.

Received by the editors July 1, 1985 and, in revised form, October 1, 1985.

1980 Mathematics Subject Classification (1985 Revision). Primary 13A15, 13B20, 13C15, 13 H99.

Key words and phrases. Analytically irreducible local ring, analytically normal local ring, $A^{*}(I)$, equivalent topologies, $E(I)$, going down, Noetherian ring, symbolic power of a prime ideal.

'The author's research on this paper was partially supported by the National Science Foundation, the first author under grant DMS 88401304, and the second under grant MCS 8301248-01. 
(vi) Let $T$ be a faïthfully flat Noetherian extension ring of $R$. If $Q \in E(I T)$ then $Q \cap R \in E(I)$. If $P \in E(I)$ and if $Q$ is a prime minimal over $P T$, then $Q \cap R=P$ and $Q \in E(I T)$.

(vii) Let $X_{1}, \ldots, X_{n}$ be indeterminates. Then

$$
E\left(I R\left[X_{1}, \ldots, X_{n}\right]\right)=\left\{P R\left[X_{1}, \ldots, X_{n}\right] \mid P \in E(I)\right\} .
$$

ProOF. (i) through (vi) follows from $[4,3.3 .1,3.2,3.3 .4,3.3 .2,3.9$, and 3.7]. For (vii), one containment follows from (vi). For the other, assume that $Q \in$ $E\left(I R\left[X_{1}, \ldots, X_{n}\right]\right)$, and that $Q \cap R=P$. By (vi), $P \in E(I)$. By (i), $Q$ is a prime divisor of $I^{m} R\left[X_{1}, \ldots, X_{n}\right]$, for large $m$. It is well known $[5,18.11]$ that this implies $Q=P R\left[X_{1}, \ldots, X_{n}\right]$, proving (vii).

We come to our main result.

THEOREM 1.2. Let $P \subset Q$ be primes in $R$. Concerning the following three statements, (a) $\Rightarrow$ (b) $\Rightarrow$ (c).

(a) $Q \in E(P)$.

(b) For some $k \geq 1, P^{(m)} \nsubseteq Q^{(k)}$ for all $m \geq 1$.

(c) There is a prime $q$ with $P \subset q \subseteq Q$, and $q \in E(P)$.

ProOF. (a) $\Rightarrow$ (b). By Lemma 1.1(ii), we have $P^{m}:\langle Q\rangle \nsubseteq Q^{(k)}$ for some $k \geq 1$, and all $m \geq 1$. However, $P^{m}:\langle Q\rangle \subseteq P^{(m)}:\langle Q\rangle \subseteq P^{(m)}$, the last inclusion since $P^{(m)}$ is $P$-primary, and $Q$ properly contains $P$. It follows that (b) holds.

(b) $\Rightarrow($ c). We will prove the contrapositive. For this, we may localize at $Q$. Thus we will assume that $(R, Q)$ is local, and that $E(P)=\{P\}$. For any $k \geq 1$, we seek an $m$ with $P^{(m)} \subset Q^{(k)}=Q^{k}$. Let $S=R-P$. If $z \in$ Ass $R^{*}$, let $q_{z}$ be a prime minimal over $P R^{*}+z$. By Lemma 1.1(iii) and (vi), $q_{z} \cap R \in E(P)=\{P\}$. Thus $q_{z} \cap S=\varnothing$ and $z \cap S=\varnothing$. The first of these shows that $P R_{S}^{*}+z_{S} \subseteq\left(q_{z}\right)_{S} \neq R_{S}^{*}$ for all $z \in$ Ass $R^{*}$. The Krull Intersection Theorem shows that $\bigcap\left\{P^{n} R_{S}^{*} \mid n \geq 1\right\}=0$. However, since $z \cap S=\varnothing$ for all $z \in$ Ass $R^{*}, S$ consists of regular elements of $R^{*}$, and $R^{*}$ is embedded in $R_{S}^{*}$. Therefore $\bigcap\left\{P^{n} R_{S}^{*} \cap R^{*} \mid n \geq 1\right\}=0$. Because $R^{*}$ is complete, for $k \geq 1$, there is an $m$ with $P^{m} R_{S}^{*} \cap R^{*} \subseteq Q^{* k}[5,30.1]$. Obviously, $P^{(m)} \subseteq P^{m} R_{S}^{*} \cap R$, and of course $Q^{* k} \cap R=Q^{k}$. Therefore, $P^{(m)} \subseteq Q^{k}$, as desired.

COROLlaRY 1.3. Let $P \subset Q$ be primes in $R$, and suppose that no prime properly between $P$ and $Q$ is an $A^{*}(P)$. Then $Q \notin E(P)$ if and only if for all $k \geq 1$ there is an $m$ such that $P^{(m)} \subseteq Q^{(k)}$. In particular, this equivalence holds whenever height $Q / P=1$.

ProOF. If $Q \notin E(P)$, then the hypothesis and Lemma 1.1(i) show that statement (c) of Theorem 1.2 is false. Thus (b) is false, giving one direction of our equivalence. The other direction is by the contrapositive of $(a) \Rightarrow(b)$ of that theorem. The second statement is immediate from the first.

For comparison to Corollary 1.3, we present the next result. (The converse of this fails.)

Proposition 1.4. Let $P \subset Q$ be primes in $R$, and suppose that no prime properly between $P$ and $Q$ is in $A^{*}(P)$. If $Q \notin A^{*}(P)$, then for all large $k, P^{(k)} \subset$ $Q^{(k)}$. (This holds for all but finitely many primes $Q$ such that height $Q / P=1$.)

Proof. Suppose $Q \notin A^{*}(P)$. Let $k$ be large enough that $A^{*}(P)=\operatorname{Ass} R / P^{k}$. The hypothesis shows that $P_{Q}$ is the only prime divisor of $P_{Q}^{k}$, so that $P_{Q}^{(k)}=P_{Q}^{k}$. 
As $P_{Q}^{k} \subset Q_{Q}^{k}$, we get $P^{(k)} \subset Q^{(k)}$. (The last statement is by the finiteness of $A^{*}(P)$.)

In $[\mathbf{7}]$, Schenzel shows that $E(P)=\{P\}$ if and only if the $P$-adic and $P$-symbolic topologies are equivalent. (That is, if and only if for all $k \geq 1$ there is an $m$ with $P^{(m)} \subseteq P^{k}$.) We get this as a corollary to Theorem 1.2.

COROLlary 1.5. Let $P$ be a prime in $R$. Then $E(P)=\{\Gamma\}$ if and only if the $P$-adic and $P$-symbolic topologies are equivalent.

Proof. Suppose $E(P)=\{P\}$. By Theorem 1.2(b) $\Rightarrow(\mathrm{c})$, if $P \subseteq Q \in \operatorname{Spec} R$, then for all $h \geq 1$, there is an $n$ with $P^{(m)} \subseteq Q^{(h)}$. Applying this to the primes $Q$ involved in a primary decomposition of $P^{k}$ (any $\left.k \geq 1\right)$, it is easy to find an $m$ with $P^{(m)} \subseteq P^{k}$. The converse is easy, using Theorem $1.2(\mathrm{a}) \Rightarrow(\mathrm{b})$, and the fact that $P^{k} \subset Q^{k} \subseteq Q^{(k)}$, for any prime $Q \supset P$.

REMARK. It is easy to see that the following three statements are equivalent for a prime $P$ in $R$. In this paper, we will only refer to the first of them. (i) The $P$-adic and $P$-symbolic topologies are equivalent. (ii) If $P \subseteq Q \in \operatorname{Spec} R$, and if $k \geq 1$, then there is an $m$ with $P^{(n)} \subseteq Q^{k}$. (iii) If $P \subseteq Q \in \operatorname{Spec} R$, and if $k \geq 1$, then there is an $m$ with $P^{(m)} \subseteq Q^{(k)}$.

REMARK. The $P$-adic and $P$-symbolic topologies are called linearly equivalent if there is an $h \geq 0$ such that $P^{(n)} \subseteq P^{n-h}$ for all $n \geq h$. This was first studied in [7]. In [6] (and [3]) it is shown to occur exactly when $U(P)=\{P\}$. Here, $U(I)=\left\{Q \cap R \mid Q \in E\left(t^{-1} \mathbf{R}(I)\right)\right\}$, with $\mathbf{R}(I)=R\left[t^{-1}, I t\right]$ the Rees ring of $R$ with respect to $I$.

2. Going down. Recall that primes $P \subseteq Q$ in a domain $R$ satisfy going down if for any integral extension domain $T$ of $R$, and $q \in \operatorname{Spec} T$ with $q \cap R=Q$, there is a $p \in \operatorname{Spec} T$ with $p \subseteq q$ and $p \cap R=P$. [2, Corollary 9.2] shows that if $P$ is prime in a Noetherian domain, and if $\bar{A}^{*}(P)=\{P\}$, then $P \subseteq Q$ satisfies going down for all primes $Q$ containing $P$. We give a new proof of this which also shows that the result remains true if $\bar{A}^{*}(P)=\{P\}$ is replaced by $E(P)=\{P\}$. (In the next section, we will discuss hypotheses under which $E(P)=\{P\}$, so that in those situations, going down will automatically hold.) In this section, all rings will be domains.

DEFINITION. Let $I$ be an ideal in the domain $R$. $M(I)=\{q \cap R \mid q$ is a prime minimal over $I T$, for some integral extension domain $T$ of $R\}$.

Our next easy lemma follows directly from $[1$, exercise $37(\mathrm{i}) \Leftrightarrow(\mathrm{ii}), \S 1.6]$. (It does not need $R$ to be Noetherian.)

LEMMA 2.1. If $P \in \operatorname{Spec} R$, with $R$ a domain, then $P \subseteq Q$ satisfies going down for all primes $Q$ which contain $P$ if and only if $M(P)=\{P\}$.

LEMMA 2.2. If $R$ is a Noetherian domain, then in the definition of $M(I)$, we may assume that $T=R^{\prime}$. Alternately, we may assume that $T=R[x]$ for some $x \in R^{\prime}$.

ProOF. Starting with $q$ and $T$ as in the definition, by lying over and incomparability, we may assume that $T=T^{\prime}$. Since $R^{\prime} \subseteq T^{\prime}$ satisfies going down, we may assume that $T=R^{\prime}$. Since only finitely many primes of $R^{\prime}$ lie over $q \cap R[\mathbf{5}, 33.10]$, pick $x$ in $q$ but in no other prime of $R^{\prime}$ lying over $q \cap R$. Then $q$ is the only prime 
of $R^{\prime}$ lying over $q \cap^{\prime} R[x]$, and so by lying over and going up, we may assume that $T=R[x]$.

The next lemma, while easy, is our key observation in this section.

LEMMA 2.3. Let $I$ be an ideal in a Noetherian domain. Then $M(I) \subseteq E(I) \cap$ $A^{*}(I) \cap \bar{A}^{*}(I)$.

Proof. Say $Q \in M(I)$. By Lemma 2.2, there is a finite integral extension domain $T$ of $R$, and $q$ minimal over $I T$ with $q \cap R=Q$. The definitions of $E(I T)$ and $\bar{A}^{*}(I T)$ make it clear that they contain all primes minimal over $I T$. Thus $q \in E(I T) \cap \bar{A}^{*}(I T)$. The result follows from Lemma $1(\mathrm{v})$ and (i), and [2, Proposition 3.22].

COROLlaRY 2.4. Let $P$ be a prime in a Noetherian domain. If either $A^{*}(P)=$ $\{P\}$, or $\bar{A}^{*}(P)=\{P\}$, or $E(P)=\{P\}$, then $P \subseteq Q$ satisfies going down for all primes $Q$ containing $P$.

ProOF. This is immediate from Lemmas 2.1 and 2.3.

3. $E(P)=\{P\}$.

LEMMA 3.1. If $(R, M)$ is such that the completion $R^{*}$ contains a single prime divisor of zero, $z$, and if $I$ is an ideal of $R$, then $M \in E(I)$ if and only if $M$ is minimal over $I$.

ProOF. Suppose $I \subseteq P \subset M$, with $P$ prime. By $[5,18.11]$ there is a prime $p$ in $R^{*}$ lying over $P$. Thus $I R^{*} \subseteq p$. Also, by its uniqueness, $z \subseteq p$. Thus $M^{*}$ is not minimal over $I R^{*}+z$, and so $M \notin E(I)$. The converse follows from the definition of $E(I)$.

Recall that the local domain $(R, M)$ is analytically irreducible if its completion is a domain (so in this case, Lemma 3.1 applies). Also, $(R, M)$ is analytically normal means that the completion is a normal domain.

LEMMA 3.2. Let I be an ideal in the Noetherian domain $R$. Suppose there is a finite integral extension domain $A$ of $R$ such that for all $Q \in \operatorname{Spec} A$ with $I \subset Q \cap R$, $A_{Q}^{*}$ has a single prime divisor of zero. Then $M(I)=E(I)=\{Q \cap R \mid Q$ is minimal over $I A\}$. In particular, if $R$ is a domain finitely generated over a field, then for any ideal $I, M(I)=E(I)=\left\{Q \cap R \mid Q\right.$ is minimal over $\left.I R^{\prime}\right\}$.

Proof. Clearly, $\{Q \cap R \mid Q$ is minimal over $I A\} \subseteq M(I) \subseteq E(I)$ (by Lemma 2.3). Suppose $P \in E(I)$. If $P=I$, then clearly $P$ is in the first set. Thus, suppose that $I \subset P$. By Lemma 1.1(v), there is a $Q \in E(I A)$ with $Q \cap R=P$. By Lemma 3.1, $Q$ is minimal over $I A$. This shows our three sets are equal. As for the final statement, for a domain $R$ finitely generated over a field, it is well known that $R_{Q}^{\prime}$ is analytically irreducible for all $Q \in \operatorname{Spec} R^{\prime}$, so that $R^{\prime}$ satisfies the hypothesis on $A$.

PROPOSITION 3.3. Let $P$ be a prime in a Noetherian domain $R$, and consider the following four statements.

(a) $E(P)=\{P\}$.

(b) The $P$-adic and $P$-symbolic topologies are equivalent. 
(c) any prime minimal over $P R^{\prime}$ lies over $P$.

(d) For all primes $Q$ containing $P, P \subseteq Q$ satisfies going down. Then

(i) (a) $\Leftrightarrow$ (b), (c) $\Leftrightarrow$ (d), and (a) $\Rightarrow$ (d).

(ii) If $R$ is finitely generated over a field, then these statements are all equivalent.

(iii) If $R$ is finitely generated over a field, if height $P=h$, and if $P$ is the radical of an ideal generated by $h$ elements, then these statements are all true.

(iv) If $R$ is locally analytically irreducible, then these statements are all true.

(v) If for every maximal ideal $M$ of $R, R_{M}$ is analytically irreducible, then these statements are all true.

(vi) If $R$ is a finite integral extension domain of $L\left[X_{1}, \ldots, X_{n}\right]$, with $L$ an analytically irreducible local domain and $X_{1}, \ldots, X_{n}$ indeterminates, and if $P$ is the radical of $p R$ for a depth 1 prime $p$ of $L$, then these statements are all true.

Proof. (i) Corollary 1.5 gives $(\mathrm{a}) \Leftrightarrow(\mathrm{b})$. Lemmas 2.1 and 2.2 give $(\mathrm{c}) \Leftrightarrow(\mathrm{d})$. Corollary 2.4 gives $(\mathrm{a}) \Rightarrow(\mathrm{d})$.

(ii) Lemma 3.2 gives $(\mathrm{a}) \Leftrightarrow(\mathrm{c})$. The rest follows from (i).

(iii) Suppose that height $P=h$ and $P$ is the radical of $\left(x_{1}, \ldots, x_{h}\right) R$. We will show that (c) holds for $P$. Let $Q$ be minimal over $P R^{\prime}$. (Note that $R^{\prime}$ is a finite $R$-module [5, 36.6].) Since $P$ is the radical of $\left(x_{1}, \ldots, x_{h}\right) R$, it is easily seen that $Q$ is also minimal over $\left(x_{1}, \ldots, x_{h}\right) R^{\prime}$. From this, it follows that height $Q \leq h$. Now $[5,34.8]$ shows

$$
\text { height } Q \cap R=\text { height } Q \leq h .
$$

However, $P \subseteq Q \cap R$, and height $P=h$, so $P=Q \cap R$. Thus (c) holds. By (ii), all of the statements hold.

(iv) By (i), it will suffice to show that (a) holds. However, Lemma 3.1 shows that no prime properly containing $P$ can be in $E(P)$, and so (a) does hold.

(v) By (iv), it will suffice to show that $R$ is locally analytically irreducible. Let $Q$ be a prime in $R$, and let $M$ be a maximal prime containing $Q$. Let $q$ be a prime minimal over $Q R_{M}^{*}$. Since $\left(R_{M}^{*}\right) / p$ is complete for every prime $p$ in $R_{M}^{*},[5,32.1]$ shows that $R_{M}^{*}$ is pseudo-geometric, as is $\left(R_{M}^{*}\right)_{q}$, using $[5,36.1]$. As $\left(R_{M}^{*}\right)_{q}$ is of finite type over $R_{M}^{*},[5,37.8]$ shows that $\left(R_{M}^{*}\right)_{q}$ is analytically irreducible. Finally, by $[5,19.2(3)], R_{Q}$ is a subspace of $\left(R_{M}^{*}\right)_{q}$, and so $R_{Q}$ is analytically irreducible. Thus $R$ is locally analytically irreducible.

(v) Since $L$ is analytically irreducible and $\operatorname{depth} p=1$, Lemma 3.1 shows that $E(p)=\{p\}$. Let $q=p L\left[X_{1}, \ldots, X_{n}\right]$. By Lemma 1 (vii), $E(q)=\{q\}$. Since $\operatorname{Rad} p R=P$, Lemma $1.1(\mathrm{v})$ and incomparability show $E(p R)=\{P\}$. Finally, since $\operatorname{Rad} p R=P$, the definition easily shows that $E(P)=E(p R)=\{P\}$. Thus (a) holds. By (i), all the statements hold.

\section{REFERENCES}

1. I. Kaplansky, Commutative ring theory, Univ. of Chicago Press, Chicago, 1974.

2. S. McAdam, Asymptotic prime divisors, Lecture Notes in Math., vol. 1023, Springer-Verlag, New York, 1983.

3. - Filtrations, Rees rings, and ideal transforms, J. Pure Appl. Algebra (to appear).

4. S. McAdam and L. J. Ratliff, Jr., Essential sequences, J. Algebra 95 (1985), 217-235.

5. M. Nagata, Local rings, Interscience Tracts 13, Interscience, New York, 1962. 
6. L. J. Katliff, Jr., The topology determined by symbolic powers of primary ideals, Comm. Algebra (to appear).

7. P. Schenzel, Symbolic powers of prime ideals and their topology, Proc. Amer. Math. Soc. 93 (1985), 15-20.

Department of Mathematics, University of TeXas, Austin, TeXas 78712

DEPARTMENT OF MATHEMATICS, UNIVERSITY OF CALIFORNIA, RIVERSIDE, CALIFORNIA 92521 